\title{
Study on Quantitative Evaluation Methods for Safety of Railway Signalling Systems
}

\section{Ikuo WATANABE}

Senior Researcher, Laboratory Head, Signalling Systems, Senior Researcher, Laboratory Head, Train Control Systems,

\section{Koji IWATA}

Researcher, Train Control Systems, Signalling \& Telecommunications Technology Div.

\begin{abstract}
We present a new safety analysis method for railway signaling systems and introduce component fault occurrence probability vector, functional failure probability vector, correlation matrix between functional failures and component faults, mitigating matrix, and several other factors. By using these factors, we can estimate the risk of signalling systems, the effectiveness of safety measures, dangerous system failure probability and other related values. We also introduce a new automatic train control system and safety technologies, and evaluate the effectiveness of these technologies by calculating the elements of mitigating matrix.
\end{abstract}

Keywords : railway signalling, safety analysis, risk analysis, fail-safe

\section{Introduction}

Safety and reliability are required for railway signalling systems because a failure of equipment may directly cause fatal accidents. To ensure safety, systems are configured based on the fail-safe principle, so that safety is maintained even when a fault has occurred in a system. The fundamental system configuration is based on a mechanism to control outputs safely in abnormal cases by designing redundant hardware and software and adding technologies to detect various errors or diagnosis technologies related thereto.

To evaluate the effectiveness of these safety technologies, we propose a method for safety analysis, which can quantitatively calculate both the risk in the railway signalling systems where various safety technologies are introduced and the effectiveness of these technologies ${ }^{12)}$. This evaluation method will efficiently accomplish the safety of railway signalling systems.

In this report, we present an integrated method to analyze and evaluate the safety of systems, describe the Automatic Train Control (ATC) system, which detects train locations and safely controls the interval between trains, and introduce various safety technologies built in this system. Finally, we apply this method to calculate and evaluate the effectiveness of these safety technologies quantitatively, taking into consideration the period of diagnosis and the size of circuits in an actual system.

\section{Integrated method for system safety analysis and evaluation}

The integrated method for system safety analysis and evaluation can represent the relationship between failure modes and safety technologies in system components, and evaluate the effectiveness of applied safety measures.

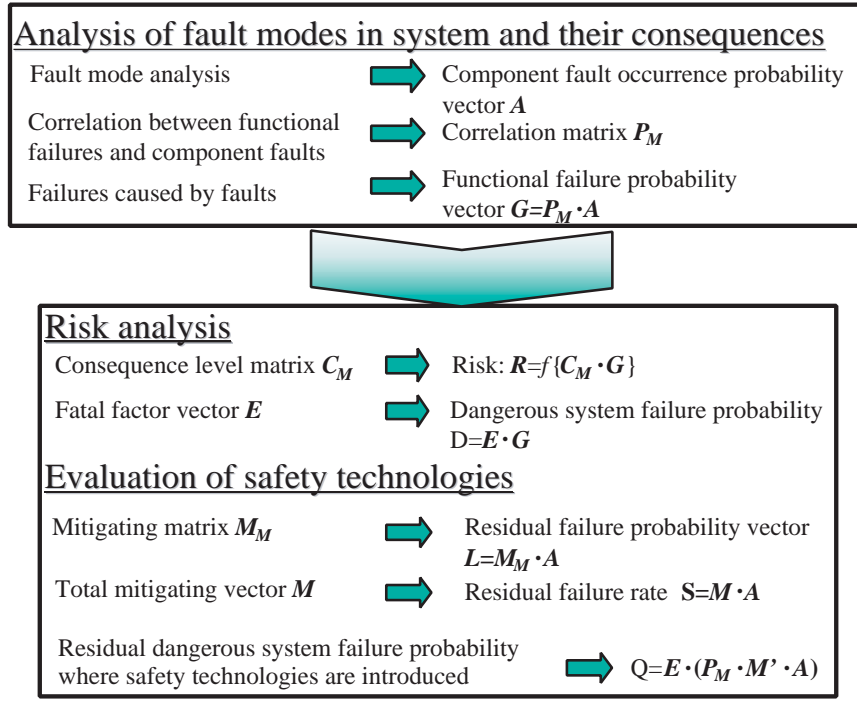

Fig. 1 Integrated method for system safety analysis and safety evaluation

This method systematically calculates various evaluation indexes on safety, such as risk and dangerous system failure rates. Figure 1 shows the flow of safety analysis by this method, which is composed of the analyses of faults in a system and their consequences, risk analysis, and evaluation of safety technologies. The risk analysis and the evaluation of safety technologies are based on vectors and matrices, which are defined by analyzing faults in a system and their consequences.

\subsection{Analysis of faults in system and their consequences}

The basic vectors and matrices in this method are as follows:

(1) Component fault occurrence probability vector of system component $(\boldsymbol{A})$ 
The fault occurrence probability of each system component is represented by a vector $(\boldsymbol{A})$.

(2) Correlation matrix between functional failures and component faults $\left(\boldsymbol{P}_{\boldsymbol{M}}\right)$

The correlation between functional system output and each component of vector $(\boldsymbol{A})$, which represents the fault occurrence probability, is shown by a value from 1 to 0 . The matrix $\left(\boldsymbol{P}_{\boldsymbol{M}}\right)$ is given by these results.

(3) Functional failure probability vector $(\boldsymbol{G})$

As shown by Equation (1), the product of the correlation matrix $\left(\boldsymbol{P}_{\boldsymbol{M}}\right)$ and the component fault occurrence probability vector $(\boldsymbol{A})$ gives a vector which shows the probability that functional outputs from the target system are wrong because of some faults. This vector is named the functional failure probability vector $(\boldsymbol{G})$.

$G=P_{M} \cdot A$

\subsection{Risk analysis and evaluation of safety technologies}

\subsubsection{Calculation of system risk level}

The functional outputs from systems cannot be considered to be the same from the viewpoint of safety. When the consequences caused by failures of each functional output element are considered, a diagonal matrix is given. This matrix is named the consequence level matrix $\left(\boldsymbol{C}_{\boldsymbol{M}}\right)$. The product of the consequence level matrix $\left(\boldsymbol{C}_{\boldsymbol{M}}\right)$ and the functional failure probability vector $(\boldsymbol{G})$ gives a vector. Each element of this vector means the product of the failure probability of each functional output and the consequence level. Therefore, when this value is compared with the reference value and a level is assigned, the risk level caused by a functional output failure is given. Since the method to quantify the consequence level is ambiguous, there are no established standards even though there are various proposals on risk analysis. Therefore, the level assignment will be denoted just by a conversion with evaluation function (f) in this report, as shown by Equation (2). In the analyzing phase, this equation will depend on the standard to be applied. The Vector $(\boldsymbol{R})$, given as a result of the conversion with the evaluation function (f), is named the risk vector.

$$
\boldsymbol{R}=\mathbf{f}\left\{\boldsymbol{C}_{M} \boldsymbol{G}\right\}
$$

The risk level of each functional output corresponds to the element $r_{j}$ of the risk vector $(\boldsymbol{R})$. Since SRL, which means the risk level of a whole system, can be considered to be the maximum value of elements $r_{j}$ of the risk vector $(\boldsymbol{R})$ in this case, it can be calculated by using Equation $(3)$

$\mathrm{SRL}=\operatorname{Max}\left\{\mathrm{r}_{\mathrm{j}}\right\}$

2.2.2 Calculation of dangerous system failure probability

The elements of the functional failure probability vector $(\boldsymbol{G})$ correspond to failure rates of functional outputs. Therefore, when only fatal elements, which are closely related with safety, are extracted from these elements, the sum of these means the dangerous system failure probability. For this calculation, the fatal factor vector $(\boldsymbol{E})$ is defined before the dangerous system failure prob- ability (D) is calculated by Equation (4), which is composed of the functional failure probability vector $(\boldsymbol{G})$ and the fatal factor vector $(\boldsymbol{E})$. In addition, the fatal factor vector $(\boldsymbol{E})$ is a vector in which functional fatal elements of a system related to safety are set at 1 and others are set at 0 .

$\mathrm{D}=\boldsymbol{E} \cdot \boldsymbol{G}$

\subsubsection{Analysis of effectiveness of safety technologies}

In order to evaluate the effectiveness of the safety technologies, it is necessary to consider what degree of a consequence caused by the fault of an element in the component fault occurrence probability vector $(\boldsymbol{A})$ is mitigated by each safety technology. Even if the applied technology is not sufficient, as long as a fault can be detected, it may be considered possible to protect the transition against the failures of functional outputs. To show the effectiveness of mitigation, the mitigating matrix $\left(\boldsymbol{M}_{\boldsymbol{M}}\right)$ is defined, which means a correlation between each row of safety technologies and each column expressing functional elements. That is, each row of the mitigating matrix $\left(\boldsymbol{M}_{\boldsymbol{M}}\right)$ corresponds to a safety technology, and its effectiveness is assigned with a value from 0 to 1 . The value is set at 0 , if a fault shown in the vector $(\boldsymbol{A})$ is protected completely, or at 1, if it can't work for mitigation. As shown by Equation (5), a calculation output, the product of the component fault occurrence probability vector $(\boldsymbol{A})$ and the mitigating matrix $\left(\boldsymbol{M}_{\boldsymbol{M}}\right)$, means a residual failure probability of system in which each safety technology is applied. This is named the residual failure probability vector $(\boldsymbol{L})$. Therefore, each element of the residual failure probability vector $(\boldsymbol{L})$ shows how effectively each safety technology can work for the system. As the value of vector $(\boldsymbol{L})$ decreases, it can work effectively.

$L=M_{M} \cdot A$

\subsubsection{Effectiveness of safety technologies}

The residual failure rate (S) of a system, where various safety technologies are combined, is given by multiplying the total mitigating vector $(\boldsymbol{M})$ shown in Equation (6), and the component fault occurrence probability vector $(\boldsymbol{A})$. Each element of vector $(\boldsymbol{M})$ is calculated as a product of all elements in each column of the mitigating matrix $\left(\boldsymbol{M}_{\boldsymbol{M}}\right)$.

$\mathrm{S}=\boldsymbol{M} \cdot \boldsymbol{A}$

2.2.5 Calculation of residual dangerous system failure probability where safety technologies are introduced

The residual dangerous system failure probability where safety technologies are introduced $(Q)$ is calculated by Equation (7), in which $\boldsymbol{M}^{\prime}$ is given by diagonalizing the total mitigating vector $(\boldsymbol{M})$.

$$
\begin{aligned}
\mathrm{Q}= & E \cdot\left(P_{M} \cdot M^{\prime} \cdot A\right) \\
& E \cdot\left(P_{M} \cdot A^{\prime}\right) \\
& E \cdot G^{\prime} \cdots \cdots
\end{aligned}
$$

The following explains what this equation means according to the calculation process, where $\boldsymbol{A}^{\prime}$ is the residual 
component fault occurrence probability vector and $G^{\prime}$ is the functional failure probability vector of a system where various safety technologies are introduced. Equation (7) is transformed into Equation (8). Equation (8) means that fatal failures are selected from the elements of the functional failure probability vector $(\boldsymbol{G})$, which cannot be detected and remains even if safety technologies are introduced in a system. By comparing ( $Q$ ) with (D), the effectiveness of mitigating dangerous system failures when safety technologies are introduced is calculated.

\section{Outline of the target system}

\subsection{Functions of the target system}

To evaluate the effectiveness of our methods, we select a new Automatic Train Control (ATC) which is now under development. ATC is an apparatus to prevent collisions between trains, and control the speed of the following train by detecting the location of the leading train (TD equipment).

In this system, a controller can detect trains on several track sections and transmits ATC signals to them for their speed control. The TD equipment transmits TD signals to several track circuits and checks these levels. When a train advances into a section and short-circuits the track circuit with vehicle axles, the level of the TD signal drops greatly. Using this principle, this equipment can detect the sections where trains exist. After the ATC apparatus receives the train locations from the TD equipment, it transmits ATC signals to track circuits, which indicate the authorized speed for each train and are based on the location of the leading train and route conditions at stations. Figure 2 shows the configuration of the ground equipment. In the case of jointless track circuits, ATC signals are transmitted only to the section where TD equipment can detect a train.

\subsection{Safety technologies}

The following shows the configuration of each unit and safety technologies applied in this system.

\subsubsection{Processing unit}

Figure 3 shows the basic configuration of the process-

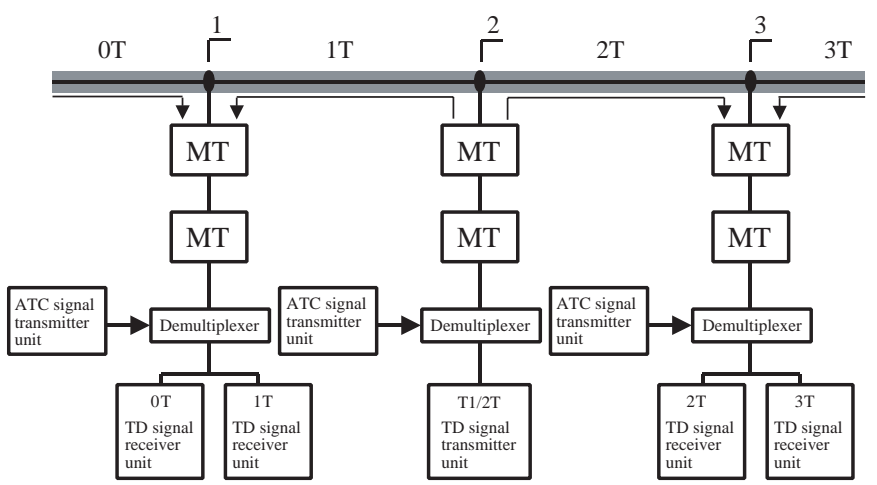

Fig. 2 Overall system of ATC

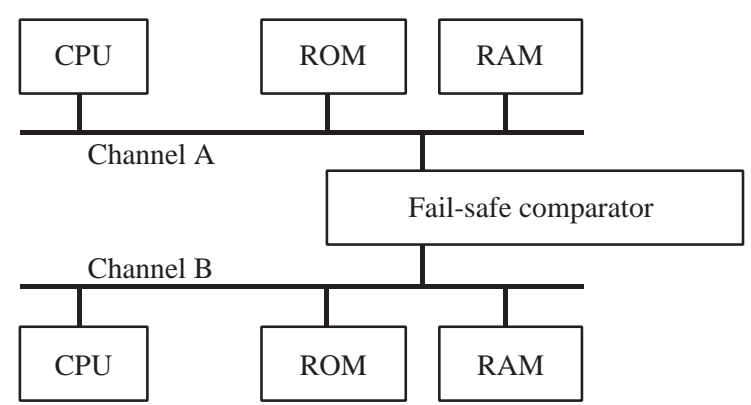

Fig. 3 Basic configuration of processing unit

ing unit. Two sets of CPU for channel A and channel B are driven synchronously at a constant designated period. In this period, all external outputs and the results of all diagnoses are compared. Details of diagnoses are as follows:

(1) Comparison of outputs in fail-safe comparator

The outputs from each CPU of channel A and channel $B$ by interruption at every designed period and every result of diagnoses are compared in the fail-safe comparator.

\section{(2) Diagnosis of ROM}

The data in ROM are read every 32 bytes in the program area and also read every two bytes in the external ROM, where the control data are recorded. These data are also compared in the fail-safe comparator.

\section{(3) Diagnosis of RAM}

The checking data are written and read every four bytes for the external RAM and every two bytes for the internal RAM.

(4) Diagnosis of register

The checking data are written and read to and from the register which can be diagnosed.

\section{(5) Diagnosis of interruption}

It is checked whether interruption occurs within the designed period. The interruption to the not-designed destination is also recognized as a fault in the process.

(6) Clock diagnosis

The clock of the CPU is checked with the clock of the oscillator.

\subsubsection{Receiver unit}

Figure 4 shows the basic configuration of a receiver unit. As shown in this Figure, the process components following the LPF are duplicated. Each result is inputted not only into the CPU of channel A but also into the CPU of channel B. The results are compared in the failsafe comparator. Even though ATT and BPF are not duplicated in these components, various safety technologies are introduced to prevent faults from malignant failures.

\subsubsection{Transmitter unit}

Figure 5 shows the basic configuration of the trans- 


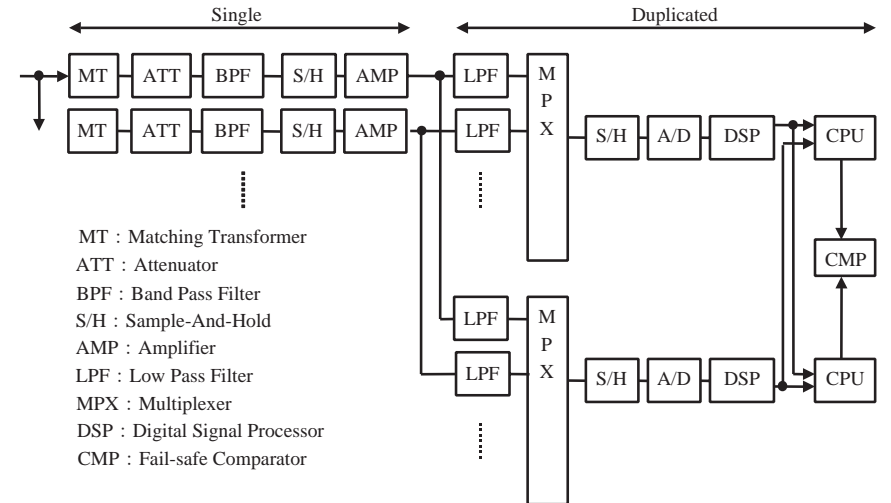

Fig. 4 Basic configuration of receiver unit

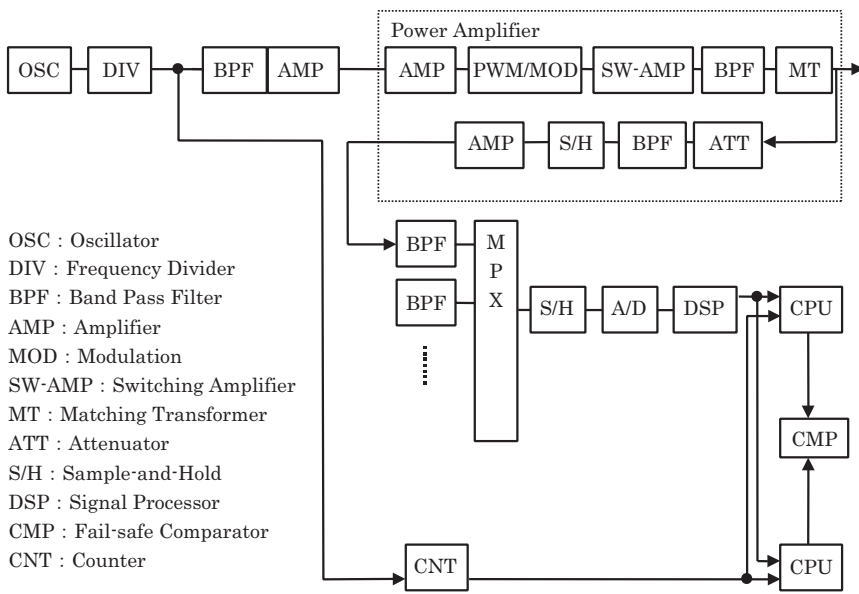

Fig. 5 Basic configuration of transmitter unit

mitter unit in the TD equipment and ATC apparatus. Although TD and ATC have only a single component, the output is diagnosed by performing a feedback check. This method can detect the malignant faults such as quasisignals generated by the frequency shift caused by faults in the oscillator or the frequency divider.

In the same way as for receiver units, safety technologies are also applied to the power amplifier unit, which consists of a single unit to protect the faults causing the malignant failure. When a fault occurs, the output levels of signals decrease and the output channel is changed to the standby unit after detecting the leveldown.

\subsubsection{Relay input}

The status of relays is inputted into each channel A/ $\mathrm{B}$, and validated in the fail-safe comparator. The breakcontact is assigned to the safety status, and the makecontact to the danger status. This make-contact is inputted in principle. To check whether the input is not fixed to the "1" status, a common line is controlled to the " 0 " status to diagnose the input circuits. Moreover, inputting both the break-contact and make-contact can be used for the safety check to use the contrariety inbetween. Figure 6 shows the basic configuration of a relay input unit.

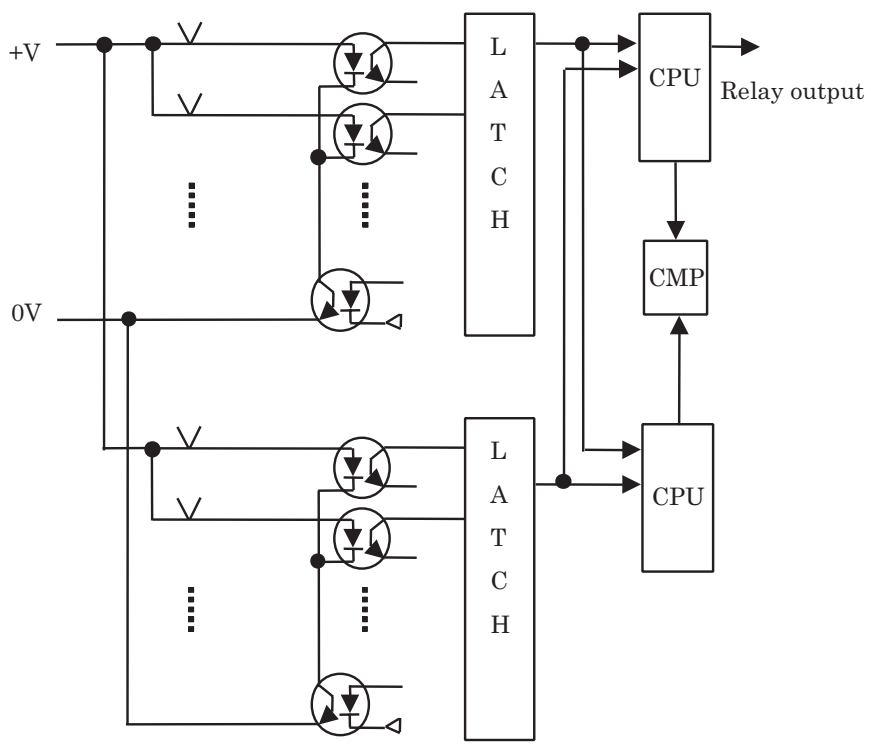

Fig. 6 Relay input

\section{Evaluation of safety technologies introduced in ATC}

The integrated method for system safety analysis and safety evaluation analyzes the risk of a system and evaluates the safety technologies with various vectors and matrices. In this chapter, we focus on some of the safety technologies introduced in ATC (Chapter 3), calculate each element $\left(\mathbf{M}_{\mathrm{Mij}}\right)$ of the mitigating matrix $\left(\boldsymbol{M}_{\boldsymbol{M}}\right)$ and evaluate the effectiveness of these safety technologies.

As described in the section 2.2.3, each row of the mitigating matrix $\left(\boldsymbol{M}_{\boldsymbol{M}}\right)$ corresponds to each safety technology and the degree of the effectiveness, which means whether a safety technology can work on each component fault occurrence probability vector $(\boldsymbol{A})$, is assigned from 0 to 1 . In this case, " 0 " means that the technology can't work, and "1" means that it can mitigate the consequence of a fault completely.

\subsection{Effectiveness of constant designed period fault diagnosis only by software}

In the processing unit, there are two ways to detect faults by various constant designed period diagnoses. One is by the process for diagnosis, and the other by the failsafe comparator, which can detect faults incidentally with the contrariety that arises by comparing the outputs from channels A and B at the time of control output. Therefore we will compare the effectiveness between two cases. One is the fault diagnosis which uses only software on the not-duplicated hardware, and the other is the fault diagnosis described in the chapter 3 , which uses both software and hardware with the fail-safe comparator. To distinguish these two cases, we call the former "fault diagnosis only by software," and the latter "fault diagnosis by the combination of software and the fail-safe comparator." In this section, the effectiveness of "fault diagnosis only by software" is discussed.

(1) Preconditions and notations

- $\lambda$ : Failure rate (constant) 
- Td : Fault diagnosis period

- To : Output period

(Fault diagnosis period is independent of the output period.)

- Cd : Detection probability of a diagnosis

( 1 : Surely detects. 0 : Loses.)

- Co : Success probability of safety control after fault detection by diagnosis

( 1 : Surely succeeds. 0 : Surely fails.)

(2) In the case of fault diagnosis period (Td) $\geqq$ output period (To)

In the case of fault diagnosis period $(\mathrm{Td}) \geqq$ output period (To), the applied diagnosis does not work from the viewpoint of time, as shown in Fig. 7. Therefore, diagnoses should be applied between a fault occurrence and an output. Since the mean time from a fault occurrence to an output is (1/2)To, the probability of diagnosis before an output is $(1 / 2)(\mathrm{To} / \mathrm{Td})$. Therefore, the probability that the diagnosis can work effectively is $(1 / 2)(\mathrm{To} / \mathrm{Td}) \mathrm{CdCo}$. The effectiveness of the fault diagnosis $\left(\mathrm{M}_{\mathrm{Mij}}\right)$, which is the probability that a fault can't be detected, is expressed by Equation (9).

$\mathrm{M}_{\mathrm{Mij}}=1-(1 / 2)(\mathrm{To} / \mathrm{Td}) \mathrm{CdCo}$

where $\mathrm{Td} \geqq$ To

As shown by Equation (9), if Td tends to infinity, $\mathrm{M}_{\mathrm{Mij}}$ tends to 1 . This result is not contradictory to the fact that there is no effectiveness without diagnoses. In addition, where $\mathrm{Cd}=1, \mathrm{Co}=1$ and $\mathrm{To}=\mathrm{Td}, \mathrm{M}_{\mathrm{Mij}}$ is 0.5 . That is, where the fault diagnosis period (Td) is longer than the output period (To), the effectiveness of mitigation against consequences caused by faults is 0.5 at most.

(3) In the case of fault diagnosis period (Td) $\leqq$ output period (To)

Where an output is made between a fault occurrence and a diagnosis, this diagnosis cannot work effectively from the viewpoint of time as shown in Fig. 8. Since the mean time from a fault occurrence to an output is $(1 / 2) \mathrm{Td}$, the probability of an output before a diagnosis is $(1 / 2)(\mathrm{Td} /$ To). On the other hand, since the probability that the diagnosis can work from the viewpoint of time is $\{1-(1 /$ $\left.2)\left(\mathrm{Td} / \mathrm{To}_{0}\right)\right\}$, the probability that a fault can be detected and an output is controlled to the safety status is $\{1-(1 /$ $2)(\mathrm{Td} / \mathrm{To})\} \mathrm{CdCo}$. Therefore, the effectiveness of safety technology $\left(\mathrm{M}_{\mathrm{Mij}}\right)$, which is the probability that a fault is overlooked, is given by Equation (10).

$\mathrm{M}_{\mathrm{Mij}}=1-\{1-(1 / 2)(\mathrm{Td} / \mathrm{To})\} \mathrm{CdCo}$

where $\mathrm{Td} \leqq$ To

As shown in Equation (10), on the condition that $\mathrm{Cd}=1$ and $\mathrm{Co}=1$, if $\mathrm{Td}$ tends to infinity, $\mathrm{M}_{\mathrm{Mij}}$ tends to 0 . This result is not contradictory to the fact that the consequence caused by a fault is negligible, if a diagnosis continues. In addition, on the condition that $\mathrm{To}=\mathrm{Td}$, both Equation (9) and Equation (10) is rewritten as Equation (11). Therefore the continuity is kept.

$\mathrm{M}_{\mathrm{Mij}}=1-(1 / 2) \mathrm{CdCo}$

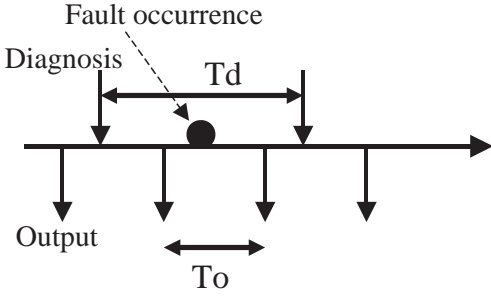

(a) Invalid diagnosis

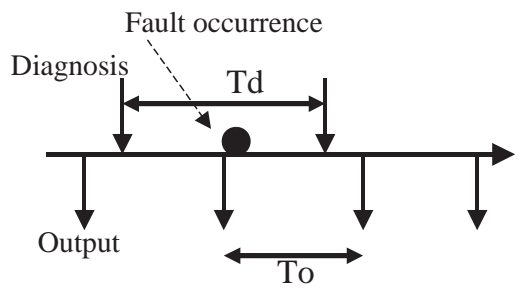

(b) Valid diagnosis

Fig. 7 Fault diagnosis ( $T d \geqq T o$ )

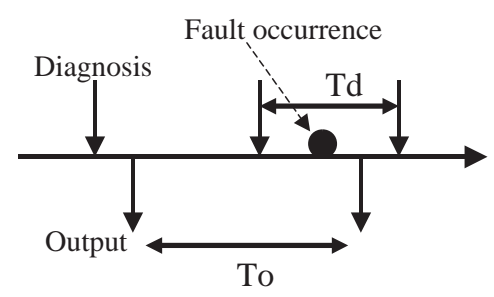

(a) Invalid diagnosis

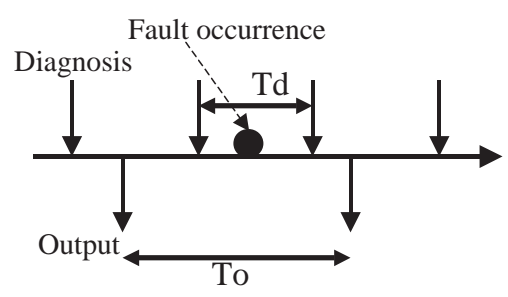

(b) Valid diagnosis

Fig. 8 Fault diagnosis ( $\mathrm{Td} \leqq \mathrm{To}$ )

\subsection{Effectiveness of constant designed period fault diagnosis by the combination of software and the fail-safe comparator}

In the case that hardware is duplicated and an output from the processor is surely diagnosed by using the fail-safe comparator before a control output, a fault can be detected, unless faults causing a malfunction get into the same failure mode between channel A and channel B. Therefore, even in an output phase, a fault diagnosis is carried out. In a normal processing phase, however, there is no guarantee that all memory areas are used within a fixed time and only the limited areas may be used. Therefore, the mitigation effectiveness of a fault consequence does not depend on the output period but depend on the fault diagnosis period. In the case of memory faults in such a structure, the effectiveness of a fault diagnosis is given by Equation (12), because a fault is detected within a diagnosis period, even if it may be kept latent in the 
normal output.

$\mathrm{M}_{\mathrm{Mij}}<\lambda \mathrm{Td}$

Where $\lambda$ is the failure rate of memory areas in each channel.

In the above-mentioned target system, since the diagnosis for all areas of RAM, which contains two M bytes, is carried out for about 2,600 (sec), where $\lambda=10^{-6}(1 / \mathrm{hr})$ and $\mathrm{Td}=2600(\mathrm{sec})=0.72(\mathrm{hr})$ in Equation (12), $\mathrm{M}_{\mathrm{Mij}}$ is less than $7.2 \times 10^{-7}$. As shown by Equation (9), in the case of using only software, $\mathrm{M}_{\mathrm{Mij}}=0.5$ at most, even if $\mathrm{Td}$ is equal to the output period. This result shows that hardware with a redundant configuration and the fail-safe comparator can play an extremely important role as a fault diagnosis for memories, for example. In addition, although the above is focused only on the memory, Equation (12) will generally be able to estimate the effectiveness of a fault diagnosis for parts in a redundant configuration and the fail-safe comparator.

\subsection{Effectiveness of the diagnosis by multiple analogue inputs}

Even in an analogue processing unit of the receiver unit, parts are also fundamentally duplicated. These outputs are also compared with each other and diagnosed. This effectiveness can be estimated by Equation (12). Where the failure rate of each part is set to $\lambda_{\text {part }}=10^{-6}$ (1/ hr) and the number of duplicated parts are about 10, the failure rate is $\lambda=\sum \lambda_{\text {part }} \fallingdotseq 10^{-5}(1 / \mathrm{hr})$. Then, when the diagnosis period $\mathrm{Td}=0.4(\mathrm{sec})=1.1 \times 10^{-4}(\mathrm{hr})$ is substituted into Equation (12), $\mathrm{M}_{\mathrm{Mij}}$ is less than $1.1 \times 10^{-9}$.

\subsection{Effectiveness of feedback check by transmitting output}

There are two kinds of diagnoses in a transmitter unit. One is the frequency check with a counter, and the other is the check by the feedback circuits of analogue signal, which has the same configuration as that of the receiver unit. These results are also compared with the reference value in each CPU of channel A/B. This method can detect the malignant faults such as quasi-signals generated by frequency shift, which arises from faults in the oscillator or the frequency divider. When the failure rate of each parts $\lambda_{\text {part }}=10^{-6}(1 / \mathrm{hr})$, and the number of parts in the frequency feedback unit is set to about 3 , the failure rate is $\lambda=\sum \lambda_{\text {part }} \fallingdotseq 3 \times 10^{-6}(1 / \mathrm{hr})$. When this result and the diagnosis period $\mathrm{Td}=0.05(\mathrm{sec})=1.4 \times 10^{-5}(\mathrm{hr})$ are set to Equation (12), $\mathrm{M}_{\mathrm{Mij}}$ is less than $4.2 \times 10^{-11}(1 / \mathrm{hr})$.

The feedback check of an analogue signal can work, only if the parts following feedback circuits are normal. This effectiveness is almost equivalent to the part failure rate in the feedback unit. When the failure rate of each part $\left(\lambda_{\text {part }}\right)$ is set to $10^{-6}(1 / \mathrm{hr})$ and the number of parts is set between 10 and $100, \mathrm{M}_{\mathrm{Mij}}$ is less than $\sum \lambda_{\text {part }} \cdot 1$ $(\mathrm{hr})=10^{-4} \sim 10^{-5}$

\subsection{Effectiveness of diagnosis of relay input}

As shown in Fig.6, in the case of the circuit configu- ration in the processing of relay inputs, it is sufficient to perform the diagnosis of inputs only once. Only if a make-contact and a break-contact are assigned to the danger state and the safety state, respectively, the diagnosis by a check pulse can detect maligant faults completely. Therefore, in the case that inputs are carried out after diagnoses, where the period between a diagnosis and an input is $\mathrm{Td}$, and the failure rate of an input circuit in a single unit is $\lambda_{\mathrm{i}}$, the effectiveness of the fault diagnosis $\left(\mathrm{M}_{\mathrm{Mij}}\right)$ in an input circuit, which means the probability that the fault can't be detected, is given by Equation (13), since this is the case where the same fault occurs in a duplicated input circuit.

$\mathrm{M}_{\mathrm{Mij}}<\left(\lambda_{\mathrm{i}} \mathrm{Td}\right)^{2}$

Where $\lambda_{\mathrm{i}}=10^{-6}(1 / \mathrm{hr})$ and $\mathrm{Td}=0.3(\mathrm{sec}), \mathrm{M}_{\mathrm{Mij}}$ is $6.9 \times 10^{-21}$.

In the case that the data are processed only at the time when a diagnosis is carried out after an input and the normality is confirmed, $\mathrm{M}_{\mathrm{Mij}}$ can be considered to be approximately 0 .

In the case where a make-contact or a break-contact is not assigned either to the danger state or to the safety state, $\mathrm{M}_{\mathrm{Mij}}$ is given by Equation (12). Where $\lambda_{\mathrm{i}}=10^{-6}(1 /$ $\mathrm{hr})$ and $\mathrm{Td}=0.3(\mathrm{sec}), \mathrm{M}_{\mathrm{Mij}}$ is $8.3 \times 10^{-11}$.

\section{Conclusion}

In this report, each element of mitigating matrix $\left(\boldsymbol{M}_{\boldsymbol{M}}\right)$, which is one of the various vectors and matrices in the integrated method for system safety analysis and safety evaluation, is calculated and the effectiveness is evaluated quantitatively with a focus placed on some safety technologies introduced into ATC. By comparing the case to use only software and that to use the redundant configuration of hardware containing a fail-safe comparator, it is made clear that the latter is extremely effective. In the future, we will calculate not only the concrete values of mitigating matrix $\left(\boldsymbol{M}_{\boldsymbol{M}}\right)$ but also those of other matrices or vectors, and continue to develop our integrated method for system safety analysis and safety evaluation.

\section{Ackowledgement}

We would like to thank Prof. Hideo Nakamura (Nihon University) and Kyosan Electric Mfg. Co., Ltd., for their contribution to this paper through their advice and valuable data on the target system.

\section{References}

1) Iwata, K., Nakamura, H.: "Unified Method for System Safety Analysis -Experimental Evaluation of a Fail-safe CPU board," Technical Report of IEICE, Vol. 99, No. 490, 1999.

2) Watababe, I., Hirao, Y., Nakamura, H., Saito, Y. "Evaluation Methods of Safety Technologies for Railway Signalling," Technical Report of IEICE, Vol. 100, No. 512,2000 